\title{
Numerical Simulation of Fog Transport in a Horizontal Channel
}

\author{
Nihel Grich ${ }^{1,4^{*}}$, Walid Foudhil ${ }^{1,3}$, Souad Harmand ${ }^{2}$, Sadok Ben Jabrallah ${ }^{1,3}$ \\ ${ }^{1}$ Laboratory of Energetics and Thermal and Mass Transfer (LETTM), Science Faculty of Tunis, \\ University of Tunis El Manar, 1060 Tunis, Tunisia. \\ ${ }^{2}$ University of Lille Nord de France, F-59000 Lille, UVHC, LAMIH, F-59313 Valenciennes, France. \\ ${ }^{3}$ University of Carthage, Sciences Faculty of Bizerte, 7021 Bizerte, Tunisia. \\ ${ }^{4}$ University of Tunis El Manar, Sciences Faculty of Tunis, Science Faculty 1060 Tunis, Tunisia.
}

\begin{abstract}
Water spraying in exchanger systems is widely used to allow cooling and improving their performance. However, transfers within the spray mixture are difficult to express because the combined mass and heat are transferred between phases, which complicates the design of the spray systems. This article presents a numerical study of the influence of water volume fraction on the distribution of the temperature in a canal. A 2D numerical model of a horizontal channel was generated and the equations governing the continuous phases (air) and the dispersed phase (water) were developed. These equations were solved using Comsol multiphysics. A comparison of the simulation results and those of the experiment reveals an acceptable concordance.
\end{abstract}

\section{Introduction}

The heat exchanger is a device that is found in several energy and thermal systems and serves to improve their efficiency and performance. It provides many functions, like phase change, heating or cooling fluid and recovery of waste heat [1]. There are different types of heat exchangers, such as the plate heat exchanger, which is widely used in industry [2]. Among the problems affecting heat exchangers is the need to increase heat transfer [3, 4]. Humidification and water spraying represent a good solution which leads to the intensification of convective transfers between the plates of the exchanger and the air. Several studies have been carried out to study this phenomenon, such as Riad et al. [5] who presented an experimental study on the air humidity effect on the external surface of a tube heat exchanger. They showed that the humidification of the air increases the coefficient of convective transfer. Other studies focus on the design of spray cooling systems. According to Wells [6], the distribution of the size of the spray droplets influences the droplet movement and the rate of evaporation. Air velocity, cone angle, injection rate, direction of injection, and metrological condition [7-12] are important parameters that affect spray cooling efficiency. In this context, several studies have been carried out on spray cooling performance $[13,14,15]$. In the present work, we are interested in a plate of a heat exchanger exposed to a fog flow, whose objective is the quantification and the optimization of the fog addition effect on the plate cooling. This article consists in modeling the problem, developing a numerical model and identifying the parameters of the model controlling the behavior of the envisaged system.

\section{Numerical approach}

To carry out the numerical simulations, it was necessary to construct the geometry, to define its discretization and to choose the mathematical and physical models describing the fluids behavior as well as the operating conditions. Numerical simulations were performed using CFD Comsol Multiphysics software. Indeed, this software uses the finite element method for the equations discretization governing the transfers.

\subsection{Geometry}

Figure 1.a represents the experimental setup. Il is a vein in which circulates an air flow controlled by a fan. The air is charged with water using a fog generator. At the end of the vein, a plate of a heat exchanger is placed. A resistance drags the heating of this plate. Indeed, thermocouples are connected in the plate to measure the temperature.

In this article, we have studied numerically the fog transport along the plate of the exchanger. We were interested in a horizontal channel whose lower wall is a metal plate of thickness " $\mathrm{e}$ " subjected to a heat flux from the bottom " q " and the upper wall is supposed adiabatic. The air enters the channel with a constant velocity and temperature where the velocity is in the turbulent regime range. The fog volume fraction and the drops diameter are considered constant. Figure 1.b shows the channel studied. The details of the plate are

\footnotetext{
Corresponding author: nihelgrichfst@gmail.com
} 
shown schematically in Figure 1.c, it is a plate composed of four layers: a plate (nylon and 30\% fiberglass), a thermal contact grease, an aluminum plate and a resistance.

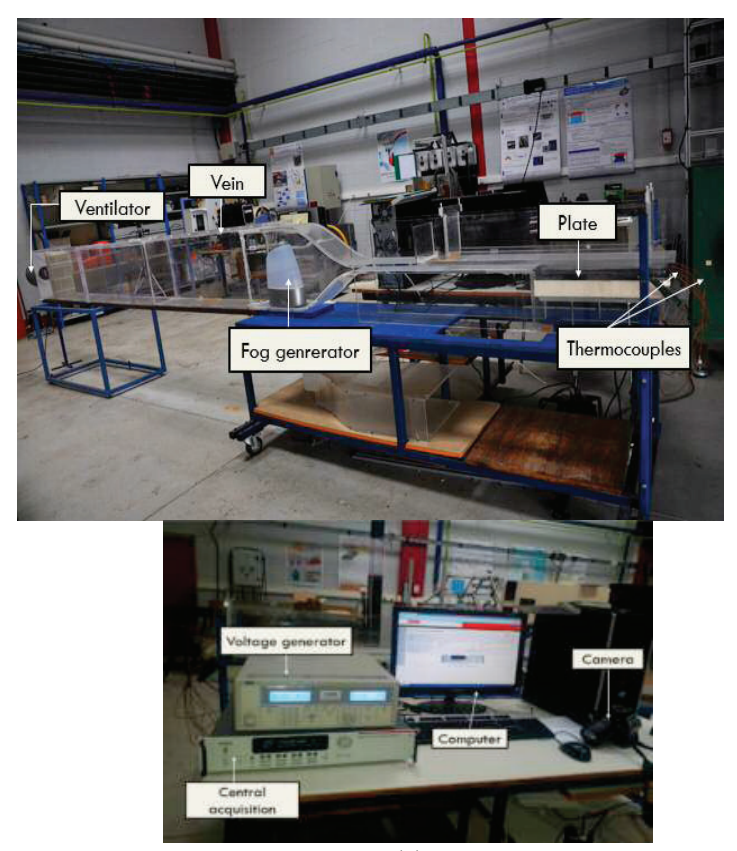

(a)

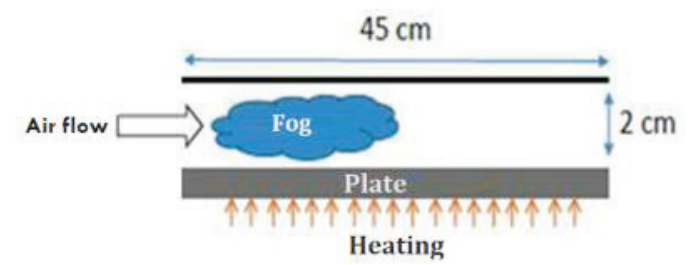

(b)

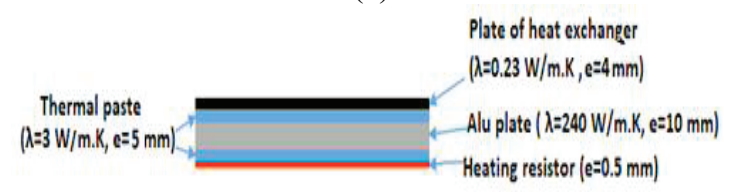

(c)

Fig. 1. Experimental setup (a), Geometry (b), Plate composition (c).

\subsection{Simplifying assumptions}

The equations describing the fog phenomenon are difficult to treat. A simplified form can be obtained with the following assumptions:

-The flow is turbulent and transient;

- Transfers are two-dimensional (2D);

- Fluids are Newtonian, incompressible;

- Air is considered a perfect gas;

- Radiation transfer is negligible;

-The fluid medium is isotropic.

\subsection{Equations}

\section{In fluid media}

The transport equations in the air as well as in the water droplets are treated as two separate phases. In fact, air is considered the continuous phase, while water represents the dispersed phase. The two coupled phases are modeled by interaction terms in the transport equations [16].

\section{Continuous phase (air)}

Mass conservation:

$$
\frac{\partial \rho}{\partial t}+\frac{\partial}{\partial x_{i}}\left(\rho u_{i}\right)=S_{m}
$$

Momentum conservation:

$$
\begin{gathered}
\frac{\partial\left(\rho u_{i j}\right.}{\partial t}+\frac{\partial}{\partial x_{i}}\left(\rho u_{i} u_{j}\right)=-\frac{\partial p}{\partial x_{j}}+\frac{\partial \tau_{i j}}{\partial x_{j}}+F_{i} \\
\text { with } \mathbf{x}_{i j}=\mu\left(\frac{\partial u_{j}}{\partial x_{i}}+\frac{\partial u_{i}}{\partial x_{j}}\right)-\frac{2}{3} \delta_{i j} \frac{\partial u_{k}}{\partial x_{k}}
\end{gathered}
$$

Energy conservation:

$$
\frac{\partial\left(\rho \mathrm{c}_{\mathrm{p} T}\right)}{\partial \mathrm{t}}+\frac{\partial}{\partial \mathrm{x}_{\mathrm{i}}}\left(\rho \mathrm{c}_{\mathrm{p}} \mathrm{u}_{\mathrm{i}} \mathrm{T}\right)=\frac{\partial}{\partial \mathrm{x}_{\mathrm{i}}}\left(\lambda \frac{\partial \mathrm{T}}{\partial \mathrm{x}_{\mathrm{i}}}\right)+\mathrm{q}_{\mathrm{m}}
$$

$\underline{\text { Species conservation }}$

$$
\frac{\partial(\rho \mathrm{C})}{\partial \mathrm{t}}+\frac{\partial}{\partial \mathrm{x}_{\mathrm{i}}}\left(\rho \mathrm{u}_{\mathrm{i}} \mathrm{C}\right)=\frac{\partial}{\partial \mathrm{x}_{\mathrm{i}}}\left(\rho \mathrm{D} \frac{\partial \mathrm{C}}{\partial \mathrm{x}_{\mathrm{i}}}\right)+\mathrm{S}_{\mathrm{m}}
$$

The parameters $\mathrm{S}_{\mathrm{m}}, \mathrm{F}_{\mathrm{i}}$ and $\mathrm{q}_{\mathrm{m}}$ are, respectively, the source terms of the mass, the momentum and the droplets energy.

\section{* Turbulence model:}

We have selected the k- $\square$ model because the flow in this study does not involve a vortex or a rotation. This two-equation model is the simplest, it has proved robust, economical and reasonably accurate for a wide range turbulent flow.

Turbulent kinetic energy equation:

$\frac{\partial\left(\rho u_{i} k\right)}{\partial x_{i}}=\frac{\partial}{\partial x_{i}}\left[\left(\mu+\frac{\mu_{t}}{\sigma_{k}}\right) \frac{\partial k}{\partial x_{i}}\right]+G_{k}-\rho \varepsilon$

Dissipation rate equation of the turbulent kinetic energy:

$$
\frac{\partial\left(p u_{i} \varepsilon\right)}{\partial x_{i}}=\frac{\theta}{\partial x_{i}}\left[\left(\mu+\frac{\mu_{i}}{\sigma_{k}}\right) \frac{\partial \varepsilon}{\partial x_{i}}\right]+C_{1 \varepsilon} G_{k} \frac{\varepsilon}{k} C_{2 \varepsilon} \rho \frac{\varepsilon^{z}}{k}
$$

Dispersed phase (droplets)

Heat and mass transfer of droplets:

The heat and mass transfer between the droplets and the air in its vicinity causes the droplet temperature change. Indeed, this is due to the heat transfer by sensible mode, which depends on convective and latent transfers.

$m_{p} c_{p} \frac{d T}{d t}=\pi d^{2} h\left(T_{a}-T_{d}\right)+\frac{d m_{p}}{d t} h_{f g}$ 
Where $h_{f g}$ is the latent heat of vaporization and $h$ is the convective heat transfer coefficient. This coefficient can be determined from the following correlation:

$$
S h_{d}=\frac{K_{e} d}{D}=2.0+0.6 R e_{d}^{0.5} S c^{0.33}
$$

The drop evaporation rate is given by:

$$
\frac{d m_{p}}{d t}=\pi d^{2} k_{c}\left(c_{s}-c_{a}\right)
$$

Where $\mathrm{k}_{\mathrm{c}}$ is the mass transfer coefficient whose value can be calculated from an empirical correlation for the droplet evaporation $[17,18]$.

$$
\mathrm{Sh}_{\mathrm{d}}=\frac{\mathrm{K}_{\mathrm{e}} \mathrm{d}}{\mathrm{D}}=2.0+0.6 \mathrm{Re}_{\mathrm{d}}^{0.5} \mathrm{Sc}^{0.33}
$$

Droplets movement:

Movement changes and thermodynamics of the dispersed phase (droplets) in the continuous phase (air) will occur due to interaction (contact or collision) with other droplets or walls. The droplets velocity variation depends on the drag force, the gravity force and the pressure force...

$\mathrm{m}_{\mathrm{p}} \frac{\mathrm{du} \mathrm{p}_{\mathrm{p}}}{\mathrm{dt}}=\mathrm{Fd}+\mathrm{Fg}+\mathrm{Fo}$

In solid media

Fourier's law ensures the heat transfer in the metal plate.

$$
\frac{\partial\left(\rho \mathrm{c}_{\mathrm{pT}}\right)}{\partial \mathrm{t}}+\frac{\partial}{\partial \mathrm{x}_{\mathrm{i}}}\left(\rho \mathrm{c}_{\mathrm{p}} \mathrm{u}_{\mathrm{i}} \mathrm{T}\right)=\frac{\partial}{\partial \mathrm{x}_{\mathrm{i}}}\left(\lambda \frac{\partial \mathrm{T}}{\partial \mathrm{x}_{\mathrm{i}}}\right)
$$

\subsection{Initial and boundary conditions}

In order to complete the problem description, it remains to provide the initial and boundary conditions. We assume that initially the computational domain is at rest with a constant and uniform velocity, temperature, and volume fraction. The hydrodynamic boundary conditions associated with this problem are characterized by the no slip of the fluid particles on the system solid walls and by its impermeability. For thermal boundary conditions, the adiabatic upper is assumed adiabatic and the lower wall (heated zone) is maintained at a constant heat flux (Figure 2).

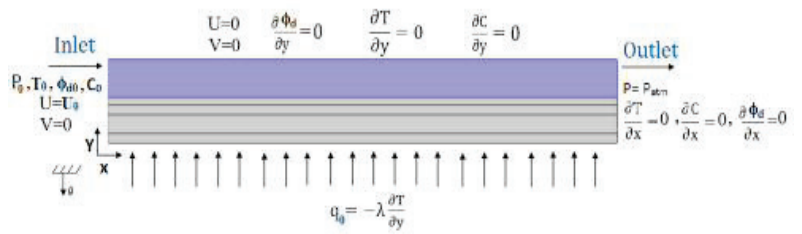

Fig.2. Boundary conditions.

\subsection{Validation}

In order to validate our numerical model, we compared the results obtained with those of the experiment (Figure 3). In this Figure, we have illustrated the temperature along the plate. From this comparison, we can note a good agreement between the numerical and experimental results. This allows to validate our numerical model.

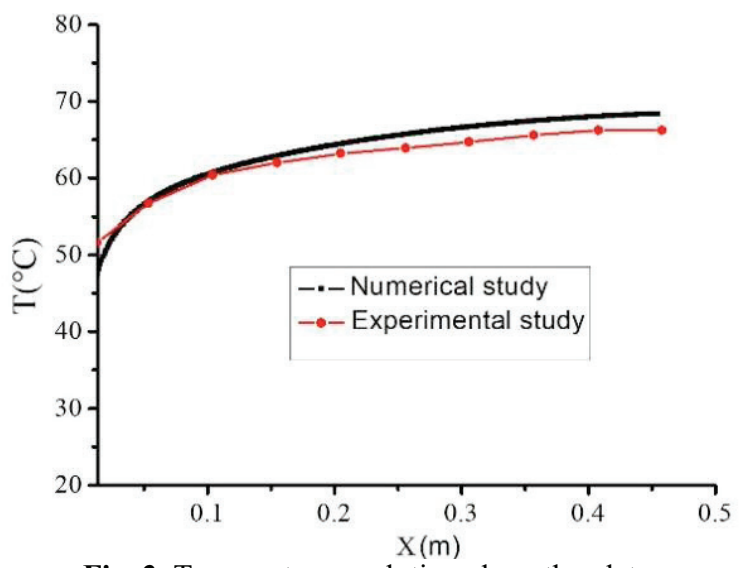

Fig. 3. Temperature evolution along the plate.

\section{Results}

The numerical simulation results present essentially the fog transport by studying the dynamic and thermal behavior along the canal. The results presented in this part are relative to the Reynolds number $\mathrm{Re}=3945$, at an ambient temperature $\mathrm{T}_{0}=20^{\circ} \mathrm{C}$ and at a heating flux of the order of $q=700 \mathrm{~W} / \mathrm{m}^{2}$.

\subsection{Dynamic field}

The Figure below shows the mixture velocity distribution (air + water) along the channel, we have traced the velocity evolution as a function of $\mathrm{Y}$ for different sections of the channel. We find that the velocity values are established and give a parabolic profile at $\mathrm{X}=0.3 \mathrm{~m}$.

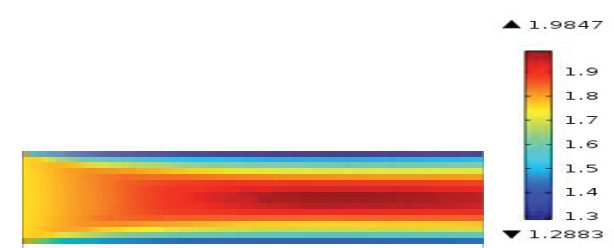

(a) 


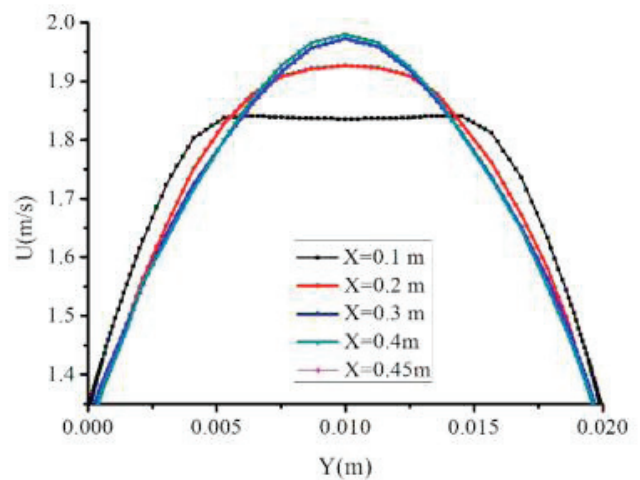

(b)

Fig.4. Velocity contour (a) and transversal velocity (b).

\subsection{Thermal field}

In Figure 5 we have illustrated the temperature contour in the studied domain. As we can see, the air is warmer at the canal outlet. This is due to the fact that the convective heat transfer between the air and the surface of the plate are the most important at the channel entrance. This also makes it cool the different solid layers and subsequently to have lower temperatures towards the leading edge.

In order to better see the distribution of the temperature on the domain, we presented in Figure 6 the temperature evolution: at the plate surface (a) and on a transversal section (b). It can be clearly seen that under the effect of convective exchanges, the interfacial temperature of the plate increases in going from the inlet to the outlet of the channel (Figure 6.a).

For the transversal representation of the temperature (Figure 6.b), it is noted that for the layers whose thermal conductivities are close, the variation of the temperature is minimal. While, for the plate, which has a low thermal conductivity, the variation is remarkable. On the other hand, for air, the temperature decreases continuously to reach its ambient value.

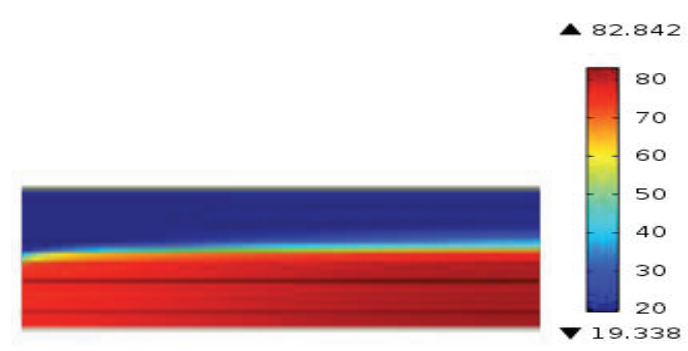

Fig.5. Temperature contour.

\subsection{Evaporation rate}

In Figure 7, we have shown the fog volume fraction contour (a), as well as the average evaporation rate along the channel (b). Indeed, this last figure shows that the evaporated rate decreases continuously going towards the channel outlet. This is explained by the fact that at the entrance of the canal the air is more loaded with water. This is confirmed by Figure 5.a, which shows that the fog volume fraction is greater at the inlet

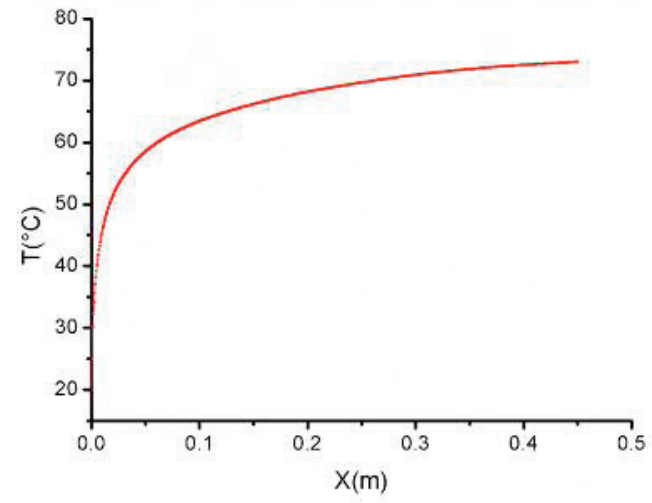

(a)

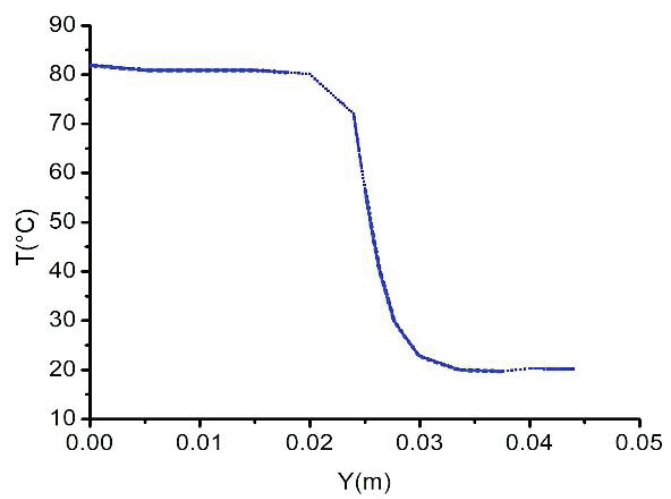

(b)

Fig. 6. Temperature evolution: at the plate surface (a) and on a transversal section (b).

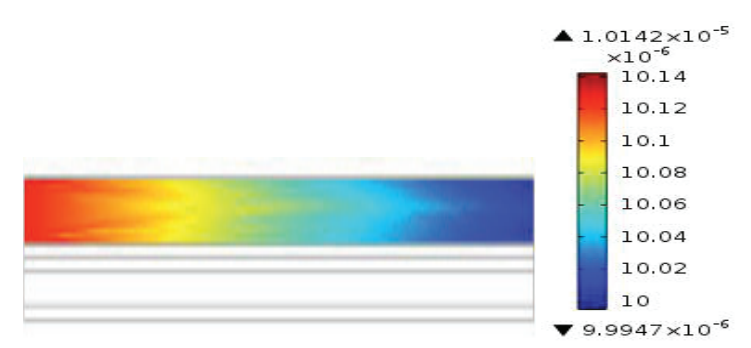

(a)

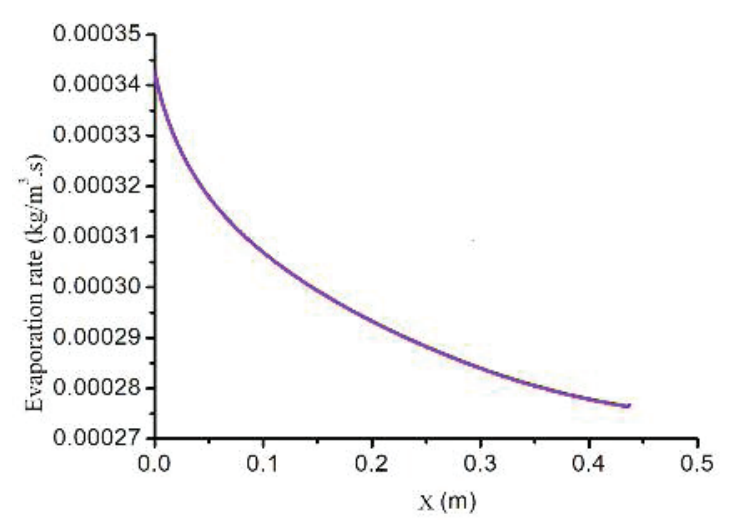

(b)

Fig. 7. Fog volume fraction contour (a) and average evaporation rate along the channel (b). 


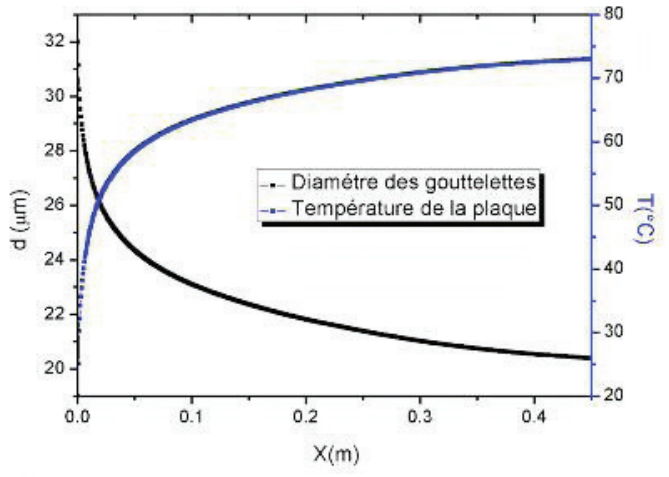

Fig. 8. Droplet diameter evolution as a function of the time.

and decreases gradually during the fog dispersion to the outlet.

Figure 8 shows the evolution of droplet diameter along the plate. This figure shows a gradual decrease in droplet volume while going towards the end of the plate. This is explained by the increase in temperature, which is used to evaporate these fine droplets distributed on the heated plate.

\subsection{Effect of fog volume fraction on the plate temperature}

We have studied the effect of fog's volume fraction on the plate temperature evolution (Figure 9). We noted that when the volume fraction increase, the interfacial temperature of the plate decrease. Indeed, an increase in the volume fraction of the fog, results in the elevation of the water content of the air injected into the channel. This makes it possible to humidify the confined environment and thus increases the convective transfers between the plate and this medium. This improvement in transfers obviously causes a decrease in the plate interfacial temperature.

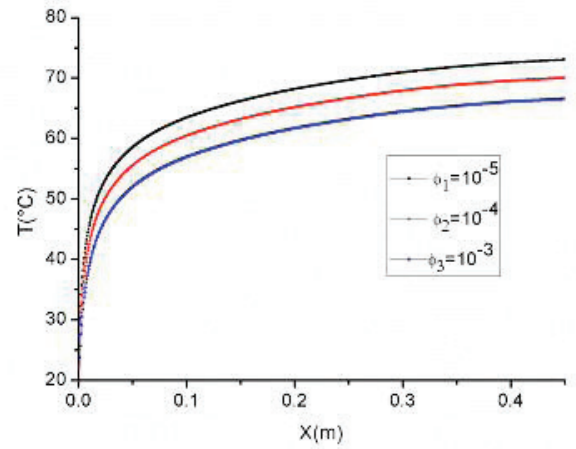

Fig. 9. Temperature evolution for different fog volume fractions.

\section{Conclusion}

In this paper, we present a two-dimensional numerical study of a case of water spray close a heat exchanger plate. The transfer processes in the airflow are modeled by the continuous phase (air) and the dispersed phase (water droplets) equations in the case of forced turbulent flow. The numerical resolution is asured by using the CFD code "Comsol Multiphysics" which uses the finite element method for the equations discretization. The presented results relate to a specific case of the operating conditions $\left(\mathrm{Re}=3945, \mathrm{~T}_{0}=20^{\circ} \mathrm{C}\right.$ and $\mathrm{q}=700 \mathrm{~W} / \mathrm{m}^{2}$ ). As well, we presented the effect of the fog volume fraction on the temperature distribution along the plate. This result shows that increasing the water content in the air decrease the exchanger plate temperature.

\section{Nomenclature}

a Vein length section, [m]

b Vein width section, [m]

C Concentration, [mol. $\left.\mathrm{m}^{-3}\right]$

$\mathrm{C}_{\mathrm{p}} \quad$ Heat capacity, $\left[\mathrm{J} \cdot \mathrm{kg}^{-1} \cdot \mathrm{K}^{-1}\right]$

$\mathrm{d}$ Diameter of the drop, $[\mathrm{m}]$

D Thermal diffusivity, $\left[\mathrm{m}^{2} / \mathrm{s}\right]$

$\mathrm{F}_{\mathrm{i}} \quad$ Droplet movement, $\left[\mathrm{kg} \cdot \mathrm{m}^{-3} \cdot \mathrm{s}^{-1}\right]$

$\mathrm{F}_{\mathrm{d}} \quad$ Drag force, [N]

$\mathrm{F}_{\mathrm{g}}$ Gravity, [N]

$\mathrm{h}$ Heat transfer coefficient, $\left[\mathrm{W} \cdot \mathrm{m}^{-2} \cdot \mathrm{K}^{-1}\right]$

$\mathrm{h}_{\mathrm{fg}} \quad$ Latent heat of vaporization, $\left[\mathrm{J}_{\mathrm{kg}} \mathrm{kg}^{-1}\right]$

$\mathrm{k}$ Turbulent kinetic energy, $\left[\mathrm{m}^{2} / \mathrm{s}\right]$

$\mathrm{k}_{\mathrm{c}} \quad$ Turbulent kinetic energy, $\left[\mathrm{m}^{2} / \mathrm{s}\right]$

m Droplet mass, [kg]

$\mathrm{Nu}$ Nusselt number, [-]

$\mathrm{P}$ Pressure, $[\mathrm{Pa}]$

q Flux de chaleur, [W.m $\left.\mathrm{m}^{-2}\right]$

$\mathrm{q}_{\mathrm{m}} \quad$ Droplet energy, [W.m- ${ }^{-3}$ ]

Re Reynolds number, [-]

Sc Schmidt number, [-]

Shd Sherwood number, [-]

$\mathrm{S}_{\mathrm{m}} \quad$ Droplet masse, [Kg. $\left.\mathrm{m}^{-3}\right]$

$\mathrm{T}$ Temperature, $[\mathrm{K}]$

t Time, [s]

$\mathrm{U}$ Velocity, $\left[\mathrm{m} \cdot \mathrm{s}^{-1}\right]$

Greek Symbols

$+\quad$ Turbulent dissipation rate, $\left[\mathrm{m}^{2} . \mathrm{s}^{-3}\right]$

$\lambda \quad$ Thermal conductivity, $\left[\mathrm{W} \cdot \mathrm{m}^{-1} \cdot \mathrm{K}^{-1}\right]$

$\mu \quad$ Dynamic viscosity, $\left[\mathrm{kg} \cdot \mathrm{m}^{-1} \cdot \mathrm{s}^{-1}\right]$

$\rho$ Density, $\left[\mathrm{kg} \cdot \mathrm{m}^{-3}\right]$

$\sigma$ Surface tension, [N.m $\left.{ }^{-1}\right]$

$\tau \quad$ Viscous stress tensor, $\left[\mathrm{kg} \cdot \mathrm{m}^{-1} \cdot \mathrm{s}^{-2}\right]$

$\phi \quad$ Volume fraction, [-]

Subscripts

a Air

$\mathrm{i}, \mathrm{j}, \mathrm{k}$ Direction indices

p Water particles

s Saturation

\section{References}

1. Bergles, E, Handbook of Heat Transfer, third edition, McGraw-Hill, New York, USA, (1998).

2. Martin, H, VDI Heat Atlas, Springer Science \& Business Media, (2010).

3. André, B, et al, in: Techniques de l'ingénieur, B2344 v1. (1995). 
4. André, B, et al, in: Techniques de l'ingénieur, B2343 v1, (1994).

5. Riad, B, et al, in: Revue Générale de Thermique, 36, 4, 289, (1997).

6. Wells, W, American Journal of Epidemiology, 20, 611 (1934).

7. Sureshkuma, R, et al, Applied Thermal Engineering, 28, 361, (2008).

8. Chaker, M, et al, Journal of Engineering for Gas Turbines and Power, 126, 545, (2004).

9. Wachtell, G. P, National Technical Information Service, (1974).

10. Tissot, $\mathrm{J}$, et al, International Journal of Thermal Sciences, 60, 23, (2012).
11. Tissot, J, et al, International Journal of Thermal Sciences, 50, 2122, (2011).

12. Chen, Z, et al, Thermal science, 22, 359, (2018).

13. Ebrahimian, V, Gorji-Bandpy, M, Thermal Science, 12, 79, (2008).

14. Zhang, Z, et al, Applied Thermal Engineering, 51, 102, (2013).

15. Vouros, A, et al, Thermal Science, 20, 149, (2016).

16. Wang, $\mathrm{T}$, et al, International Mechanical Engineering Congress and Exposition, 20, 1, (2004).

17. Ranz, W. E. and Marshall, W. R. Jr, Chem. Eng. Prog, 48, 141, (1952)

18. Ranz, W. E. and Marshall, W. R. Jr, Chem. Eng. Prog, 48, 173, (1952). 